\title{
Discussions on the reuse of items based on the delay time modelling
}

\author{
A. C. J. Santos*, C. A. V. Cavalcante** and S. Wu*** \\ * Departamento de Eng. de Produção, Universidade Federal de Pernambuco, Brazil: a.c.j.santos@random.org.br \\ ** Departamento de Eng. de Produção, Universidade Federal de Pernambuco, Brazil: cristiano@ufpe.br \\ *** Kent Business School, University of Kent, UK: $\underline{\text { s.m.wu@kent.ac.uk }}$
}

\begin{abstract}
The delay time concept has been used in a wide variety of applications in maintenance policy optimisation. In some situations, the sojourn time of an item at a defective state may hamper the performance of the item due to its reduced efficiency, and may therefore incur more repair cost. Consequently, the longer the sojourn time in a defective state, the worse the influence may cause. Despite these facts, in the literature, there is little research investigating the influence of the sojourn time in the defective state on its relevant cost. In addition, a limited number of papers study delay time modelling for the case that repaired items can be reused. This paper therefore extends a recently published paper by Santos et al. (2021) and presents some insights into the delay time modelling for second-hand items. In general, the proposed model may be applied to the scenarios where used items may be replaced by a new or refurbished one. The main contribution of this paper is on its exploration of methods of item reuse, from which new directions for further studies are established.
\end{abstract}

\section{Introduction}

The reuse of items (or second-hand items) provides an environmentally friendly way to conserve natural resources and maintain economic sustainability (Yu et al. 2021; Santos et al. 2021). Interesting papers have been recently published to explore new ways to reuse or recycle second-hand items, on which, however, replacement policies have been studied to a very limited extent in comparison with replacement policies for new items (Park et al., 2020). A recently published paper proposes a schematic framework that models the possible reuse of an item considering its defective condition, based on the delay time concept (Santos et al. 2021). This paper takes a further step by proposing a delay time model for the reuse of items, comparing it with the traditional delay time model, and then establishing the optimal time to perform inspections for a prespecified percentage of items to be reused in the long-run. The paper lays the foundation for future development of new delay time models in the context of reuse.

In more detail, this paper presents a new delay time model for the reuse of items that may have defective states. It considers that, prior to the failure, the item passes from the good state to the minor defective state (in which the item can be reused) and then to the major defective state (in which the item cannot be reused anymore). The objective is to emphasise reusing actions by minimizing relevant cost to seek the optimal inspection period.

The motivation for the development of this work comes from two different aspects. First, nowadays governments encourage firms to develop strategies and practices that make their relationship with the environment more sustainable in social, economic and environmental ways. Second, the use of items that have degraded along the time becomes very common in the industries. Some types of items, such as mechanical ones, can be generally refurbished and reused. As such, the method developed in this paper may also be applied in many different industrial sectors.

The remainder of this paper is structured as follows. Section 2 presents a possible practical context in which the proposed model may be useful. The proposed model is presented in section 3. Section 4 discusses the results and conducts sensitivity analysis. Section 5 concludes the paper.

\section{Practical context and motivation}

Maintenance policy optimization is an important research topic in operational research (Scarf, 1997). Along with the theoretical development, the practical aspect should be emphasized as well. In the real-world, there are many types of items that may wear and deteriorate. Such items can be mechanical items (Carter, 1986). In these contexts, the delay time concept is a good tool to depict the failure process of such items. 
Additionally, some items may have experienced more than one defective state prior to failure. An interesting piece of work related to the development of maintenance policies for items with two defective states can be found in $\mathrm{Wu}$ (2011) and Wang et al. (2017). This paper proposes a novel method with the delay time concept for items that can be reused and then illustrates its application in the mantle and bowl liner of cone crusher equipment, which is widely used in the mining industry. The mantle and the bowl liner may be interpreted as a component and a socket that together execute an operational function. As a result, they can be considered as a single-component system, or an item (Ascher and Feingold, 1984; Scarf and Cavalcante, 2012). Such an item is subject to a continuous wearing process due to its characteristic of operation (crushing hard material), as discussed by Sinha and Mukhopadhyay (2014). The wearing process is intrinsic and can be one of the main causes of such failures. Besides, the degradation process is also influenced by the feed material gradation that is crushed (Metso Outotec, 2021). As a result, such an item has two defective states: The first one relates to the normal wear and the second to the abnormal wear. Figure 1 illustrates a simplified view of the equipment, the item under analysis, and the two types of wear.

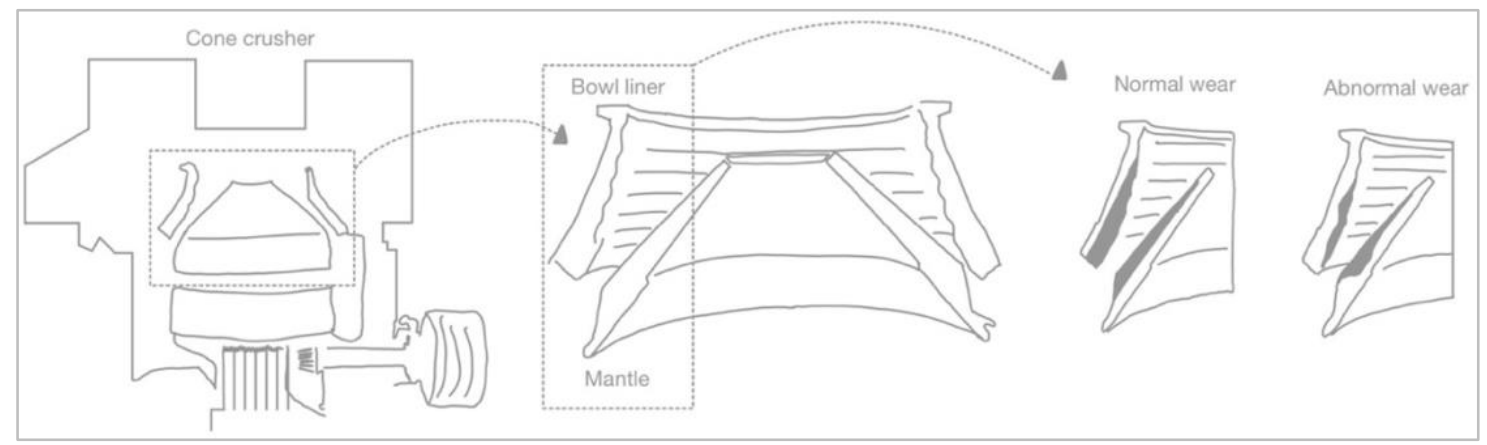

Figure 1. Illustrative example of one possible practical application. On the left, a draft of a cone crusher equipment. In the middle, the bowl liner (external element) and the mantle (internal element). On the right, representations of normal wear (considered as minor defective state) and abnormal wear (considered as major defective state). Source: Adapted from Metso Outotec (2021).

In practice, the normal wear can be considered as the minor defective state, which will not develop to a severe negative consequence. An item at this state can still be reused if the state is identified. The abnormal wear can be considered as the major defective state, which will develop to a severe consequence. An item with a major defect cannot be reused anymore. As such, the model that will be developed in this paper emphasizes the detection of the minor defective state to provide reduced maintenance cost and a more friendly environmental impact. The details about the development of the model are explained in the following section.

\section{Development of the model}

As above-mentioned, the proposed model establishes the possibility of reusing an item that is subject to twodistinct defective states. The model determines the optimal inspection interval $T$ in order to minimize the longrun cost per unit of time $C(T)$. The idea is to detect the defect in a less degraded state to reduce maintenance cost and improve environmental impact. The assumptions are defined as follows.

\subsection{Assumptions}

This paper makes the following assumptions.

1. The item has four states: good, minor defective, major defective or failed. The minor defective state does not promote severe damage to the item whereas the major defective state does.

2. Repair on the item with the minor defect brings the item to the as-good-as-new state.

3. Inspections are performed in order to detect the state of the item and to prevent non-reusing action.

4. Upon inspection, if the item is detected as minor defective, it will be reused.

5. Upon inspection, if the item is detected as major defective or failed, it will be disposed of.

6. The inspections are perfect and take place every $T$ units of time. Each inspection incurs a cost of $C_{i}$.

7. If the item cannot be reused, the repair cost consists of the acquisition cost of a new item $C_{p}$ and the disposal cost $C_{d}$. 
8. If the item can be reused, the repair cost consists of the cost to make that item available again. This cost is a function of the time in the minor defective state: $C_{r}(\tau)=w_{1} C_{p}+\mathrm{w}_{2} C_{p} \tau$.

9. If failure occurs, there exist the failure $\operatorname{cost} C_{f}$ and the disposal cost $C_{d}$.

10.The sojourn times in good state $x$, in minor defective state $y$, and in delay time $h$, are distributed according to known Weibull distributions, for which the probability density functions are $f_{x}(x), f_{y}(y)$ and $f_{h}(h)$, respectively.

Remark: In Assumption 8, $w_{1}$ and $w_{2}$ are the weights. We assume that the cost of repairing an item for reuse varies from $w_{1}$ of the cost of acquiring a new one and it changes along the time in the defective state in a way that it will not larger than the cost of acquiring a new one.

\subsection{Calculation of the decision criterion}

The decision criterion adopted is $T$ and the objective function is the long-run cost per unit of time $C(T)$. That is, we aim to minimise $C(T)$ to obtain $T$. All possible cases of the model are illustrated in Figure 2.

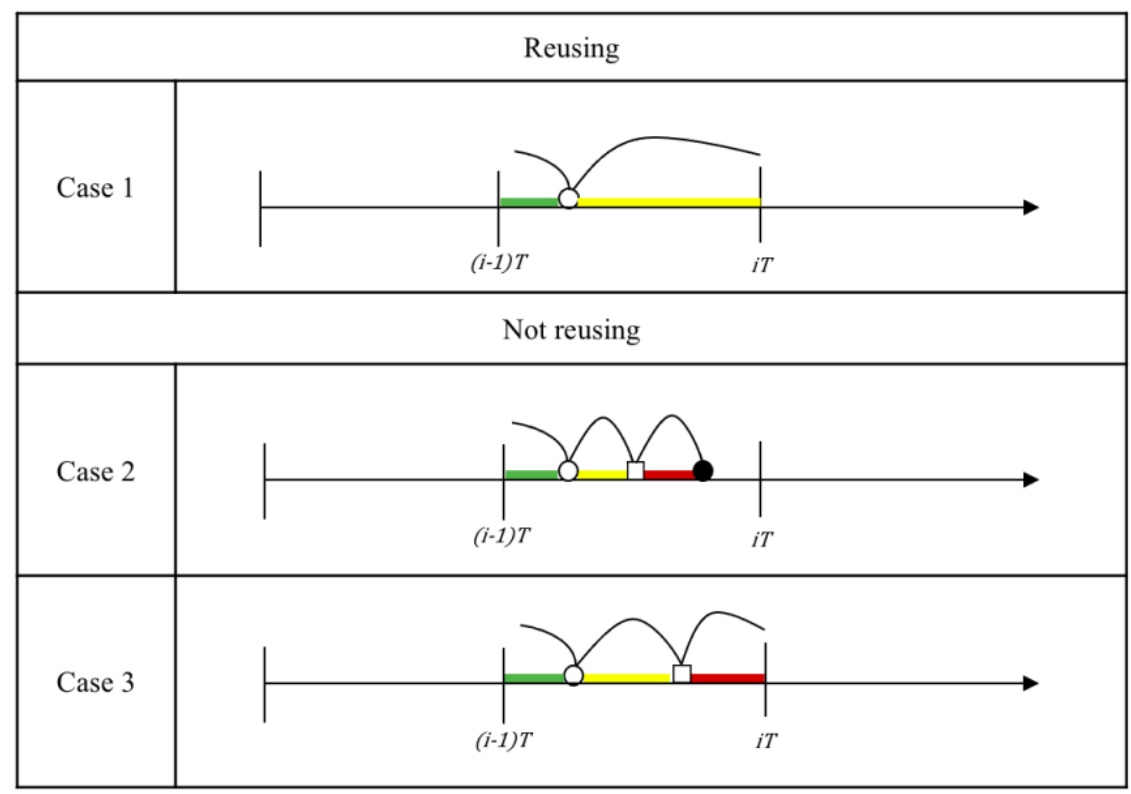

Figure 2. All possible cases.

Case 1. Reuse - The cycle ends in the minor defective state. Therefore, the item will be repaired in order to return to it later in another replacement opportunity. The probability of the minor defective state being detected at an inspection is given by equation (1). Note that for this case to happen, the defect must necessarily arrive within the interval $[(i-1) T, i T]$ which establishes the limits of the integral that represents the probability of the defect arrival within this interval $\int_{(i-1) T}^{i T} f_{x}(x) d x$. Similarly, the time in the minor defective state must be longer than $(i T-x)$, so that, this probability is $\int_{i T-x}^{\infty} f_{y}(y) d y$. The summation from 1 to $\infty$ is considered to reflect the long-run analysis by means of an infinite number of inspections. The other expressions were established using the same logic.

$P_{1}=\sum_{i=1}^{\infty} \int_{(i-1) T}^{i T} f_{x}(x) \int_{i t-x}^{\infty} f_{y}(y) d y d x$

Here there is an important point about the value of this probability. As previously commented, the reuse will happen whenever it is possible. As such, whenever the cycle ends in the minor defective state, the item will be reused. Therefore, this probability provides a good estimate about the maximum percentage of reuse. The expected cost of a cycle finishing due to the detection of a minor defective state is given by equation (2). Note that in this case the repair cost is a function of the time in the defective state $C_{r}(i T-x)$. 
$U(T)_{1}=\sum_{i=1}^{\infty} \int_{(i-1) T}^{i T} \int_{i T-x}^{\infty}\left[i C_{i}+C_{r}(i T-x)\right] f_{x}(x) f_{y}(y) d y d x$

The expected length of a cycle that finishes in the minor defective state is given by equation (3). Note that it ends in the inspection that detects the minor defect.

$V(T)_{1}=\sum_{i=1}^{\infty} \int_{(i-1) T}^{i T} \int_{i T-x}^{\infty}[i T] f_{x}(x) f_{y}(y) d y d x$

Case 2. Not reusing - The cycle ends due to a failure. Therefore, the item cannot be reused (because it is too degraded) and needs to be disposed of. The probability of case 2 is shown in equation (4).

$P_{2}=\sum_{i=1}^{\infty} \int_{(i-1) T}^{i T} f_{x}(x) \int_{0}^{i T-x} f_{y}(y) \int_{0}^{i T-x-y} f_{h}(h) d h d y d x$

The expected cost of a cycle finishing due to a failure is given by equation (5). Note that in this case there exist both the failure cost $C_{f}$ (the highest one considered in the policy) and the disposal $\operatorname{cost} C_{d}$ (related to the inconveniences to discharge the item).

$U(T)_{2}=\sum_{i=1}^{\infty}\left[(i-1) C_{i}+C_{f}+C_{d}\right] \int_{(i-1) T}^{i T} f_{x}(x) \int_{0}^{i T-x} f_{y}(y) \int_{0}^{i T-x-y} f_{h}(h) d h d y d x$

The expected length of a cycle that ends in a failure is given by equation (6). Note that it is represented by the period of time in the good, minor defective and major defective states.

$V(T)_{2}=\sum_{i=1}^{\infty} \int_{(i-1) T}^{i T} \int_{0}^{i T-x} \int_{0}^{i T-x-y}(x+y+h) f_{x}(x) f_{y}(y) f_{h}(h) d h d y d x$

Case 3. Not reusing - The cycle ends in the major defective state. Therefore, the item cannot be reused (because it is too degraded) and needs to be disposed of. The probability of case 3 is shown in equation (7).

$$
P_{3}=\sum_{i=1}^{\infty} \int_{(i-1) T}^{i T} f_{x}(x) \int_{0}^{i T-x} f_{y}(y) \int_{i T-x-y}^{\infty} f_{h}(h) d h d y d x
$$

The expected cost of a cycle that ends in the major defective state is given by equation (8). Note that in this case there exist both the replacement $\operatorname{cost} C_{p}$ and the disposal cost $C_{d}$ (related to the inconveniences to discharge the item).

$$
U(T)_{3}=\sum_{i=1}^{\infty}\left[i C_{i}+C_{p}+C_{d}\right] \int_{(i-1) T}^{i T} f_{x}(x) \int_{0}^{i T-x} f_{y}(y) \int_{i T-x-y}^{\infty} f_{h}(h) d h d y d x
$$

The expected length of a cycle that ends in the major defective state is given by equation (9). Note that it ends in the inspection that detects the major defect.

$$
V(T)_{3}=\sum_{i=1}^{\infty} \int_{(i-1) T}^{i T} \int_{0}^{i T-x} \int_{i T-x-y}^{\infty}[i T] f_{x}(x) f_{y}(y) f_{h}(h) d h d y d x
$$


Since all possible cases were defined and $\sum_{n=1}^{3} P_{n}=1$. Equation (10) shows the long-run cost per unit of time $C(T)$ and section 4 presents the discussion on the results.

$$
C(T)=\frac{\sum_{i=1}^{3} U(T)_{n}}{\sum_{i=1}^{3} V(T)_{n}}
$$

\section{Results and discussion}

This section presents the results of the model's application in a hypothetical example in which the item is subject to minor and major defective states before failure. All parameters considered are shown in Table 1, as well as the obtained optimum policy. In this section, we set $w_{1}=0.2$ and $w_{2}=0.1$. $\beta$ and $\eta$ represent, respectively, shape and scale parameters of considered Weibull distributions. This type of distribution is widely used to represent degradation processes in maintenance models.

First, we analyse the cost if the traditional approach was applied. By a traditional approach (case 0), we mean that the discharging of the item occurs regardless the item is in minor or major defective state. Second, we analyse our proposed policy of reusing items that are detected in the minor defective state (case 1). Finally, we demonstrate a short sensitivity analysis on the main important parameters for the reusing policy (cases 29).

Table 1 . Results and sensitivity analysis.

\begin{tabular}{ccccccccccccccc}
\hline & \multicolumn{1}{c}{ Distribution parameters } & \multicolumn{1}{c}{ Cost parameters } & \multicolumn{2}{c}{ Optimum policy } \\
\hline & Cases & $\beta_{x}$ & $\eta_{x}$ & $\beta_{y}$ & $\eta_{y}$ & $\beta_{h}$ & $\eta_{h}$ & $C_{i}$ & $C_{r}$ & $C_{p}$ & $C_{d}$ & $C_{f}$ & $T$ & $C(T)$ \\
\hline Not reusing & 0 & 3 & 5 & 2.5 & 1 & 2.5 & 1 & 0.05 & $C_{p}$ & 1 & 0.5 & 5 & 1.15203 & 0.35904 \\
\hline Reusing & 1 & 3 & 5 & 2.5 & 1 & 2.5 & 1 & 0.05 & $C_{r}(\tau)$ & 1 & 0.5 & 5 & 0.70523 & 0.14919 \\
\hline & 2 & 3 & 5 & 2.5 & 0.5 & 2.5 & 1 & 0.05 & $C_{r}(\tau)$ & 1 & 0.5 & 5 & 0.46663 & 0.20767 \\
& 3 & 3 & 5 & 2.5 & 1.5 & 2.5 & 1 & 0.05 & $C_{r}(\tau)$ & 1 & 0.5 & 5 & 0.90080 & 0.12620 \\
Sensitivity & 4 & 3 & 5 & 2.5 & 1 & 2.5 & 1 & 0.05 & $C_{r}(\tau)$ & 0.5 & 0.5 & 5 & 0.76953 & 0.11602 \\
analysis on & 5 & 3 & 5 & 2.5 & 1 & 2.5 & 1 & 0.05 & $C_{r}(\tau)$ & 1.5 & 0.5 & 5 & 0.65411 & 0.18091 \\
the reusing & 6 & 3 & 5 & 2.5 & 1 & 2.5 & 1 & 0.05 & $C_{r}(\tau)$ & 1 & 0.25 & 5 & 0.74764 & 0.14333 \\
policy & 7 & 3 & 5 & 2.5 & 1 & 2.5 & 1 & 0.05 & $C_{r}(\tau)$ & 1 & 0.75 & 5 & 0.67046 & 0.15440 \\
& 8 & 3 & 5 & 2.5 & 1 & 2.5 & 1 & 0.05 & $C_{r}(\tau)$ & 1 & 0.5 & 3 & 0.72268 & 0.14811 \\
& 9 & 3 & 5 & 2.5 & 1 & 2.5 & 1 & 0.05 & $C_{r}(\tau)$ & 1 & 0.5 & 7 & 0.69113 & 0.15015 \\
\hline
\end{tabular}

As shown in Table 1, the reusing policy establishes a considerable reduction of $58.5 \%$ in the long-run cost per unit of time $C(T)$ by reducing the optimal inspection interval $T$ in $38.8 \%$. Therefore, in this specific case in which the item may be reused if the degradation is up to a certain level, it is more interesting to anticipate inspection in order to detect the minor defective state rather than the major defective state.

Regarding the sensitivity analysis, the model behaves as expected. When the scale parameter of the minor defective state is higher, the model returns a longer inspection interval $T$ and provides a reduced $\operatorname{cost} C(T)$ (case 3). When the repair cost $C_{p}$, the disposal cost $C_{d}$ or the failure cost $C_{f}$ increase, $C(T)$ increases even with more frequent inspections (cases 5, 7 and 9).

Finally, the reusing policy provides a better cost allied to a better preservation of environmental resources due to the possibility of refurbishing the minor defective item to reuse it again. Figure 3 illustrates the cost benefit of reusing policy over the traditional approach. 


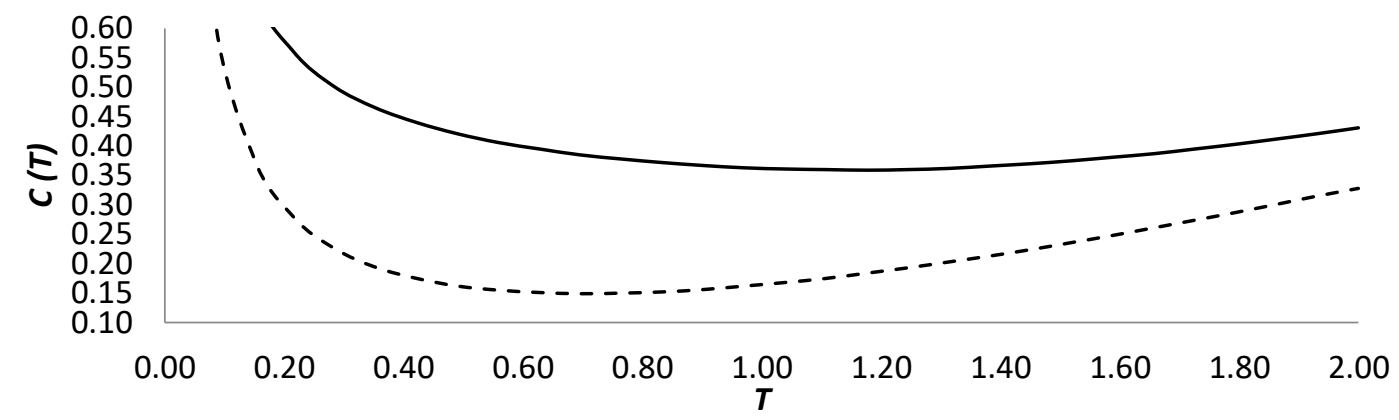

Figure 3. Long-run cost per unit of time for the traditional approach (non-reusing policy) and the proposed approach (reusing policy), respectively, solid and dashed lines.

\section{Final considerations}

The main contribution is the development of an alternative reuse model that provides both economic and environmental advantages when compared to the traditional approach used in industries. This paper proposed an idea of a maintenance policy for items that deteriorate/age and are subject to two defective states. The proposed method uses the delay time concept to model different conditions of the item and to establish the optimum inspection interval that provides the minimum long-run cost per unit of time. An interesting practical context for application was described in order to emphasize the model's applicability. A limitation is the consideration that a reused item has the same behaviour of a new one. This is a topic to be developed in future works.

\section{Acknowledgement}

This study was financed in part by the Coordenação de Aperfeiçoamento de Pessoal de Nível Superior - Brasil (CAPES) - Código de Financiamento 001. The work of Cristiano Cavalcante has been supported by CNPq (Brazilian Research Council). The work of Augusto Santos has been supported by FACEPE (Foundation for Science and Technology of the State of Pernambuco).

\section{References}

Ascher H, Feingold H (1984) Repairable Systems Reliability. New York: Marcel Dekker.

Carter ADS (1986) Mechanical Reliability. London: Macmillan.

Metso Outotec (2021) Nordberg HP series cone crushers - Wear parts application guide. Available at: $<$ https://www.mogroup.com/globalassets/saleshub/documents---episerver/application-guide-hp-series2602-en-lowres.pdf > Accessed on 28 Mar 2021.

Park M, Jung KM, Park DH (2020). Warranty cost analysis for second-hand products under a two-stage repair-or-full refund policy. Reliab. Eng. Syst. Saf. 193, 106596.

Santos ACJ, Cavalcante CAV, Ribeiro LA (2021) The use of second-hand items based on the delay time theory. Process Safety and Environmental Protection 146, 118-125.

Scarf PA (1997) On the application on mathematical models in maintenance. Eur. J. Oper. Res 99, 493-506.

Scarf PA, Cavalcante CAV (2012) Modelling quality in replacement and inspection maintenance. Int. J. Productions Economics 135, 372-381.

Sinha RS, Mukhopadhyay AK (2015) Reliability centered maintenance of cone crusher: a case study. Int $J$ Syst Assur Eng Manag 6, 32-35.

Wang H, Wang W, Peng R (2017) A two-phase inspection model for a single component system with threestage degradation. Reliab. Eng. Syst. Saf. 158, 31-40.

$\mathrm{Wu}, \mathrm{S} .$, (2011). Optimal inspection policy for three-state systems monitored by variable sample size control charts. Int. J. Adv. Manuf. Technol, 55 (5), 689-697.

Yu KH, Zhang Y, Li D, Montenegro-Marin CE, Kumar PM (2021). Environmental planning based on reduce, reuse, recycle and recover using artificial intelligence. Environmental Impact Assessment Review, 86, 106492. 